\title{
Cecal Volvulus Associated with Intestinal Malrotation Presenting as Postoperative Intestinal Obstruction
}

\author{
Arunandhichelavan Arulmolichelvan $^{\mathrm{a}}$ Arjun Sivaraman ${ }^{\mathrm{a}}$ Ashok Muthukrishnan ${ }^{\mathrm{b}}$ \\ Departments of General Surgery, ${ }^{a}$ Kasturba Medical College, Manipal University, Manipal, and ${ }^{b}$ PSG Medical College, \\ Coimbatore, India
}

\section{Key Words}

Intestinal volvulus $\cdot$ Intestinal obstruction $\cdot$ Intestinal disease $\cdot$ Congenital malformation $\cdot$ Malrotation of the gut

\begin{abstract}
Objective: To report a case of intestinal malrotation predisposing to cecal volvulus following nonabdominal surgery in an adult. Presentation and Intervention: A 23-year-old male with known anorectal malformation developed a postoperative intestinal obstruction following percutaneous nephrolithotomy for left renal calculus. Computed tomography of the abdomen revealed a grossly dilated cecum $(9 \mathrm{~cm})$ with ileocecal junction on the left side and small bowel loops on the right side of the abdomen. The patient underwent emergency exploratory laparotomy and retroperitonealization cecopexy. Bowel functions were normal at the followups. Conclusion: This case highlights the importance of suspecting cecal volvulus even following nonabdominal surgery in the presence of predisposing factors.
\end{abstract}

Copyright $\odot 2012$ S. Karger AG, Basel

\section{Introduction}

Cecal volvulus is a rare entity with incidence ranging from 2.8 to 7.1 per million people per year. It is responsible for $1-1.5 \%$ of all adult intestinal obstructions and
$25-40 \%$ of all volvulus involving the colon [1] and is one of the modes of presentation of intestinal malrotation and abnormal fixation that commonly presents in early childhood. Cecal volvulus presenting as intestinal obstruction in an adult case of malrotation is rare. It is associated with significant morbidity and mortality because of the diagnostic delay as a result of rarity of the condition and altered anatomy [2]. Treatment and prognosis depends largely upon the viability of the dilated cecum. Surgery is the only definite treatment of the cecal volvulus.

\section{Case Report}

A 23-year-old male with known anorectal malformation underwent PCNL for left pelvic calculus. He complained of abdominal pain and distension associated with bilious vomiting and obstipation on the second postoperative day. Upon examination, the left upper abdomen was grossly distended with visible intestinal loops. Plain X-ray of the abdomen in erect posture showed a dilated large bowel loop in the left hypochondrium with two air fluid levels and the rectal gas shadow was not seen (fig. 1b). Emergency computed tomography (CT) of the abdomen (fig. 1a) showed the cecum located in the midline dilated with feces measuring up to a diameter of $9 \mathrm{~cm}$. The ileocecal junction was present on the left side; most of the small bowel was on the right side. An emergency explorative laparotomy was performed. The cecum, located at the midline with the duodeno-jejunal flexure to the right of the superior mesenteric vessels and the small bowels pushed to the right side, was grossly dilated and twisted along the

\section{KARGER}

Fax +4161306 1234

E-Mail karger@karger.ch

www.karger.com
(C) 2012 S. Karger AG, Basel

$1011-7571 / 12 / 0214-0389 \$ 38.00 / 0$

Accessible online at:

www.karger.com/mpp
Dr. Arjun Sivaraman, MS

Kasturba Medical College

Manipal, Karnataka (India)

Tel. +919008760687

E-Mail journalsubmission2010@gmail.com 
Fig. 1. a CT scan showing the dilated cecum with twisted ileocecal junction (arrow) on the left side. $\mathbf{b}$ X-ray of the abdomen. Erect view showing dilated bowel in the left upper abdomen with two air fluid levels.

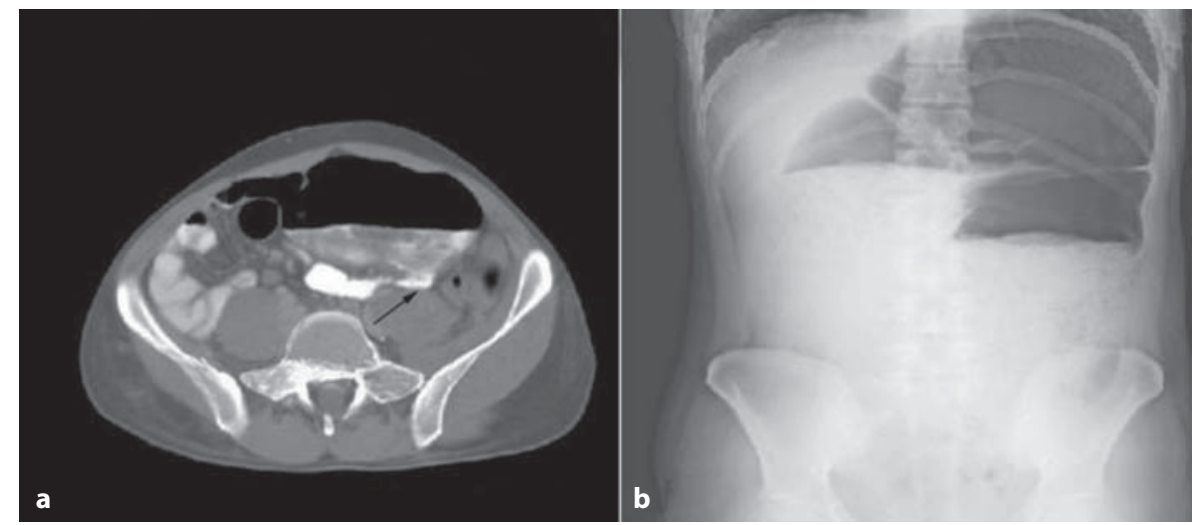

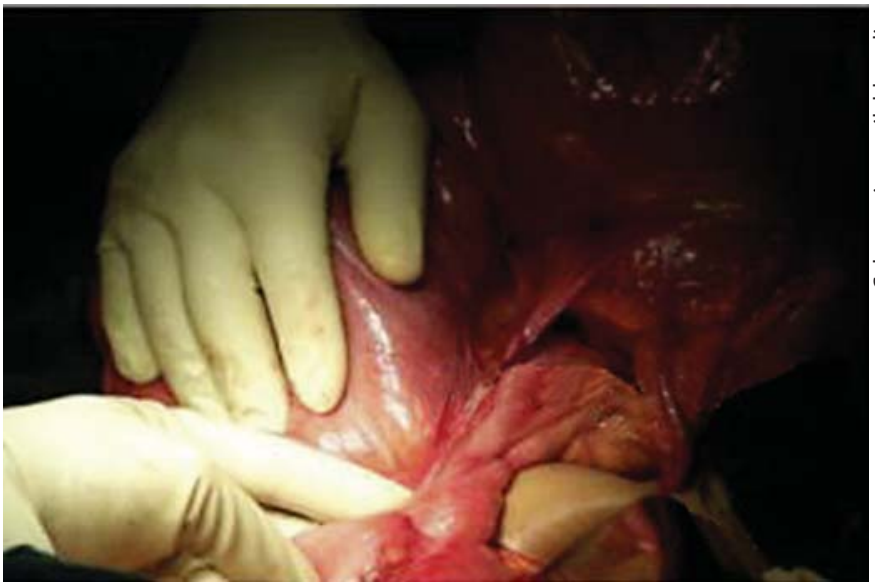

Fig. 2. Intraoperative picture showing dilated cecum with twisted ileocecal junction on the left side.

ileocolic artery (fig. 2). Since there was no evidence of compromise to the vasculature, detorsion and retroperitonealization cecopexy was performed. The postoperative period was uneventful: the patient had a prolonged recovery period without complications. Follow-up after a month revealed normal status.

\section{Discussion}

Cecal volvulus is a rare cause of large bowel obstruction, second only to sigmoid volvulus in its frequency of occurrence of large bowel volvulus. The main pathogenesis of cecal volvulus may be attributed to the long right colonic mesentery. Although retroperitoneal fixation of the cecum and ascending colon fails in $10-15 \%$ of the population, cecal volvulus remains rare [3]. Common causes are usually idiopathic or congenital bands. The other precipitating factors includes the causes that impair the evacuation of the colon due to stenotic process, postoperative paralytic ileus, pregnancy with cecal dislocation, abdominal surgery requiring mobilization of the right colon and high residual diet.

Cecal volvulus is one of the known modes of presentation of intestinal malrotation. Mid-gut malrotation has been estimated to occur in approximately one in 500 live births [4]. Approximately $85 \%$ of malrotation cases present in the first 2 weeks of life [5]. In adulthood, intestinal malrotation is estimated to occur in $0.2-0.5 \%[6,7]$. A literature review by von Flue et al. [8] cites 40 cases from 1923 to 1992.

Since both cecal volvulus and intestinal malrotation are rare, diagnostic delay is common and hence complications occur. The possible etiologies of cecal volvulus in the patient of this report are malrotation and abnormal fixation of bowel and possible paralytic ileus following general anesthesia. Although the patient had a history of anorectal malformation, cecal volvulus was never considered as a possible cause of the intestinal obstruction. However, there was no diagnostic delay and imaging was done at the earliest sign of altered postoperative course.

Investigations commenced with plain X-ray of the abdomen, which usually shows a dilated large bowel segment with air fluid levels. Abdominal CT has become necessary for accurate pre-operative diagnosis of intestinal volvulus. The 'whirl' sign, intestinal twisting, and the clockwise or counter-clockwise direction of the volvulus have been well described in the radiology literature [9]. A CT scan will point out other associated abnormalities and features of malrotation such as the abnormal relationship between the superior mesenteric artery (SMA) and superior mesenteric vein (SMV) and pancreatic un- 
derdevelopment or absence of the uncinate process, etc. can be achieved.

Surgery remains the main definite treatment for cecal volvulus. The best long-term results have been with resection and primary anastomosis. Isolated reports of emergency laparoscopic cecopexy have been described more recently [10].

The patient in our study had the classic whirlpool sign and transposition of superior mesenteric artery to the right of superior mesenteric vein on CT scan (fig. 2). Because there was no compromise of bowel vascularity and no associated anomalies, we performed a cecopexy.

\section{Conclusion}

This case clearly illustrated the importance of suspecting cecal volvulus even following nonabdominal surgery.

\section{References}

1 Rabinovici R, Simansky DA, Kaplan O: Cecal volvulus. Dis Colon Rectum 1990;33: 765-769.

12 Rogers RL, Harford FJ: Mobile cecum syndrome. Dis Colon Rectum 1984;27:399-402.

$\checkmark 3$ Samuel M, Boddy SA, Nicholls E: Large bowel volvulus in childhood. Aust NZ J Surg 2000;70:258-262.

-4 Torres AM, Ziegler MM: Malrotation of the intestine. World J Surg 1993;17:326-331.
5 McIntosh R, Donovan EJ: Disturbances of rotation of the intestinal tract. Am J Dis Child 1939;56:116-166.

6 Frantzides CT, Cziperle DJ, Soergel K, Stewart E: Laparoscopic Ladd procedure and cecopexy in the treatment of malrotation beyond the neonatal period. Laparosc Endosc Percutan Tech 1996;6:73-75.

7 Mazziotti MV, Strasberg SM, Langer JC: Intestinal rotation abnormalities without volvulus: the role of laparoscopy. J Am Coll Surg 1997; 185:172-176.
$>8$ Von Flue M, Herzog U, Ackermann C: Acute and chronic presentation of intestinal nonrotation in adults. Dis Colon Rectum 1994; 37:192-198.

$\checkmark 9$ Delabrousse E, Sarlieve P, Sailley N: Cecal Volvulus: CT findings and correlation with pathophysiology. Emerg Radiol 2007;14: 411-415.

10 Madiba TE, Thomson SR: The management of cecal volvulus. Dis Colon Rectum 2002; 45:264-267. 